\title{
CENÁRIO da ASSISTÊNCIA EM SAÚdE MENTAL/USO de SUbSTÂNCIAS PSICOATIVAS na Região de saúde de Piracicaba, São Paulo, Brasil
}

Iris Renata Vinha ${ }^{1}$

O consumo de álcool e outras drogas tem representado problema emergente no Brasil. Este estudo objetivou caracterizar a assistência a dependentes químicos da Região de Saúde de Piracicaba e, para tal, realizou-se levantamento de dados do Sistema de Informação do Ministério da Saúde (DATASUS). Constatou-se que os gastos aumentaram, aproximadamente, $100 \%$ desde 2003 no local de estudo, principalmente nas cidades com hospital psiquiátrico (Araras e Rio Claro). Embora se saiba que as internações por transtornos mentais representem uma parcela dos casos existentes, esses dados podem servir para programação e gestão da atenção em saúde mental.

Descritores: Unidade Hospitalar de Psiquiatria; Saúde Mental; Transtornos Relacionados ao Uso de Substância; Avaliação de Serviços de Saúde.

\section{Health Care Setting in Mental health /Psychoactive substance use in the Piracicaba Health Area, São Paulo, Brazil}

The use of alcohol and other drugs has been a problem in Brazil. The purpose of this study was to characterize the health care delivered to Drug Users in the Piracicaba Health Area. To do this, a survey was performed on the Health Ministry Information System (DATASUS). It was found that costs increased approximately 100\% since 2003 at the study location, especially in cities with psychiatric hospitals (Araras and Rio Claro). Although it is known that hospitalizations due to Mental Disorders account for only a part of the exiting cases, these data may serve to program and manage mental health care.

Descriptors: Psychiatric Department, Hospital; Mental Health; Substance-Related Disorders; Health Services Evaluation.

\footnotetext{
1-Enfermeira, Especialista em Administração em Saúde e em Administração Hospitalar e de Sistemas de Saúde, Hospital das Clínicas, Faculdade de Medicina, Universidade de São Paulo/EASP-Fundação Getúlio Vargas; Especialista em Enfermagem Psiquiátrica e Saúde Mental - Escola de Enfermagem de Ribeirão Preto, Universidade de São Paulo, SP, Brasil; Especialista em Dependência Química Universidade Federal de São Paulo, SP, Brasil. E-mail: irisvinha@hotmail.com
} 


\section{ESCENARIO DE LA ATENCIÓN EN SALUD MENTAL / USO DE SUSTANCIAS psicoactivas en la Región Sanitaria de Piracicaba, São Paulo, Brasil}

El consumo de alcohol y otras drogas ha representado un problema emergente en Brasil. Este estudio objetivó caracterizar la atención a Dependientes Químicos de la Región Sanitaria de Piracicaba, para lo cual se realizó recopilación de datos del Sistema de Información del Ministerio de Salud (DATASUS). Se constató que los gastos aumentaron aproximadamente el 100\% desde 2003 en la región estudiada, principalmente en las ciudades con hospital psiquiátrico (Araras y Rio Claro). Aunque se sabe que las internaciones por Transtornos Mentales representan apenas una parte de los casos existentes, tales datos pueden servir para la programación y gestión de la atención en salud mental.

Descriptores: Servicio de Psiquiatría en Hospital, Salud Mental; Trastornos Relacionados con Sustâncias; Evaluación de Servicios de Salud. 
O Brasil, desde as décadas de 1970 e 80, apresenta contínuos movimentos de reforma nas políticas públicas, referentes ao cuidado em saúde mental ${ }^{(1)}$.

Nesse sentido, a Reforma Psiquiátrica Brasileira se baseia na desinstitucionalização, refletida não somente no campo técnico-científico, mas também no campo social, no qual o portador de transtorno mental tem sua cidadania e liberdade garantidas ${ }^{(2)}$.

Paralelo a isso, tem-se construído outra política de saúde desde a nova constituição, principalmente com a Lei Orgânica da Saúde (8.080/1988) e a criação do Sistema Único de Saúde (SUS), tratando-se, também, de marco importante para avanços na garantia de acesso aos serviços de saúde, com envolvimento e esforço de diversos profissionais na mudança do modelo de atenção. A ideia é que se promova a descentralização hospitalar e médica e reorientação das práticas assistências, com ênfase no fortalecimento da atenção básica e na ampliação dos direitos sociais $^{(3-4)}$.

Nesses dois contextos - reorientação geral do sistema de saúde e movimento da reforma psiquiátrica-nota-se que o tratamento em saúde mental tem caminhado com base na reabilitação psicossocial. Nesse sentido, se torna possível dar ênfase ao resgate da autonomia dos portadores de transtorno mental, através da elevação do poder contratual psicológico, social e necessidade de busca do direito ao exercício da cidadania e não mais na cura, na tutela e na reeducação moral ${ }^{(5-7)}$.

Propõe-se, portanto, que sejam criados novos espaços para atenção em saúde mental, deixando de ter os hospitais psiquiátricos e as internações e as reinternações constantes como principais recursos, passando a ter como perspectiva a ampliação da rede de serviços substitutivos ao modelo manicomial e prática em Centros de Atenção Psicossocial

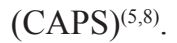

Desde 1976, com marco legal na Lei 6.368, a assistência a dependentes químicos faz parte desse processo, o que reafirmou o uso e a dependência de drogas como parte do campo da saúde, realizando modificações, principalmente no caráter de tratamento em regime hospitalar e extra-hospitalar ${ }^{(9)}$.

O consumo de álcool e de outras drogas psicoativas tem representado problema biopsicossocial emergente no Brasil e no mundo. A constatação desse grave problema de saúde pública no país encontra ressonância na sociedade, com relação comprovada entre o consumo e agravos sociais que dele decorrem ou que o reforçam. Cerca de $10 \%$ da população urbana consome abusivamente algum tipo de droga psicoativa, porcentagem na qual os brasileiros também se enquadram ${ }^{(10)}$.

A Organização Mundial de Saúde (OMS) aponta o álcool como a substância psicoativa mais consumida no mundo e também como a droga de escolha entre crianças e adolescentes, o que ocorre também no Brasil, em qualquer faixa etária. Esse consumo entre adolescentes vem aumentando, principalmente entre os mais jovens (de 12 a 15 anos de idade) e entre as meninas. Segundo o "V Levantamento Nacional com Estudantes", realizado em 2004, pelo Centro Brasileiro de Informações sobre
Drogas Psicotrópicas (CEBRID), 65,2\% dos estudantes relataram já ter feito uso de álcool em algum momento da vida, 44,3\% nos últimos 30 dias, 11,7\% fazem uso frequentemente, ou seja, seis ou mais vezes no mês e $6,7 \%$ podem ser classificados como usuários pesados, isto é, fizeram uso do álcool 20 vezes ou mais no último mês ${ }^{(11)}$.

Em um estudo da CEBRID, de base populacional, sinalizou-se que $12,3 \%$ das pessoas pesquisadas, com idade entre 12 e 65 anos, preenchem critérios para a dependência do álcool, e cerca de $75 \%$ já beberam pelo menos uma vez na vida ${ }^{(12)}$.

Além disso, o Brasil apresenta diferenças regionais marcantes, o que leva também a diferenças quanto ao tipo de droga consumida, quantidade, sexo e idade dos usuários. O consumo de cocaína concentra-se principalmente no Sudeste e no Sul do Brasil, sendo mais comum em algumas populações específicas, porém, praticamente inexistente em outras. No entanto, como já citado, o consumo de álcool parece seguir distribuição mais uniforme ${ }^{(13)}$.

No que diz respeito aos atendimentos hospitalares, provocados pelo abuso de psicotrópicos, levantamentos realizados apontam o álcool como responsável por cerca de $90 \%$ das internações por dependência. Também mostram que as internações por cocaína vêm aumentando gradativamente desde 1987. Por outro lado, as internações por maconha vêm diminuindo ${ }^{(13)}$.

Encarado o contexto brasileiro de desafios do SUS e da reforma psiquiátrica e o impacto econômico do tratamento para dependência química, no ano 2004, foi estabelecida, pelo Ministério da Saúde, a Política de Atenção Integral aos Usuários de Álcool e Outras Drogas, na qual estão traçadas diretrizes gerais, de acordo com a Lei Orgânica da Saúde (8.080/1988) e, a principal em saúde mental, a Lei Paulo Delgado $(10.216 / 2001)^{(10)}$.

Essa política tem como objetivo integrar diversos Ministérios Governamentais, uma vez que essa problemática atinge outros segmentos da sociedade, mas, também, agir no tratamento, prevenção e redução de danos, visando não somente a abstinência completa, mas o consumo reduzido.

O Programa de Atenção Integral a Usuários de Álcool e Outras Drogas especifica que os serviços para esse tipo de atendimento, no SUS, devem estar interligados e abrangem aqueles de atenção básica, Centro de Atenção Psicossocial Álcool e Drogas (CAPS-AD), ambulatórios e unidades hospitalares especializadas e de referência. A rede de suporte social (associações de ajuda mútua e entidades da sociedade civil) deve complementar a assistência $\operatorname{prestada}^{(8)}$. Serviços esses que atendem as principais diretrizes do SUS - integralidade, descentralização, hierarquização e equidade - e que vão ao encontro dos pressupostos da reforma psiquiátrica.

Nessa perspectiva, o grande desafio dos gestores municipais e estaduais é planejar os recursos e praticar esse novo modelo assistencial. Para auxiliar, um componente essencial no processo decisório é a informação que tem como principal fonte a base do Ministério da Saúde DATASUS, gratuita, de fácil acesso e constantemente alimentada pela rede pública de saúde brasileira. 


\section{Objetivo}

Este estudo teve como objetivo apresentar informações baseadas no perfil da população, no dimensionamento, na disponibilidade e na alocação financeira de serviços de saúde do banco de dados do DATASUS que representem o diagnóstico da situação de saúde e da assistência prestada a Dependentes Químicos e que auxiliem os gestores municipais da Região de Saúde de Piracicaba do Estado de São Paulo no planejamento, na tomada de decisão e na pactuação dos recursos em saúde.

\section{Material e Método}

O estudo utilizou fontes de informações secundárias, disponíveis no Sistema de Informação do Ministério da Saúde - DATASUS, como segue.

-Instituto Brasileiro de Geografia e Estatística (IBGE)

-Cadastro Nacional de Serviços de Saúde (CNES)

-Sistema de Informações Hospitalares (SIH)

-Sistema de Informações Ambulatoriais (SIA)

-Sistema de Informações sobre Mortalidade (SIM).

-Desses sistemas, foram selecionadas as informações que mostravam:

-a forma de organização da Região de Saúde por meio do número de municípios englobados - dados demográficos - IBGE;

-características básicas da população atendida por meio do número de munícipes a serem assistidos pelo sistema de saúde local - dados demográficos - IBGE;

-situação de saúde da população dependente química por meio da morbidade hospitalar e mortalidade - SIM e $\mathrm{SIH}$;

-estrutura da regional para assistência em dependência química por meio -

-da quantidade, tipo e qualidade de serviços ofertados - CNES, SIH e SIA;

-tipo de cobertura assistencial, por meio de número de leitos e indicador de rede substitutiva (CAPSAD) por habitante - IBGE, SIH e SIA;

-investimento de recursos financeiros nessa modalidade, por meio de série histórica SIH e SIA.

Os resultados filtrados foram analisados de acordo com a Lei Paulo Delgado, $\mathrm{n}^{\circ} 10.216$, de 6 de abril de 2001, baseada no processo de reforma psiquiátrica brasileira, e com a Portaria ${ }^{\circ} 2.197$, de 14 de outubro de 2004, baseada na Política de Atenção Integral aos Usuários de Álcool e Outras Drogas (2004) e na Política Nacional sobre Drogas (2005), ambas do Ministério da Saúde. A Portaria $\mathrm{n}^{\circ} 1.028 / \mathrm{GM}$, de 1 de julho de 2005, que define ações de redução de danos sociais e à saúde decorrente de uso de substâncias, não possui relação direta com o objetivo do estudo e não foi utilizada na análise. Porém, é importante lembrar que se trata de documento relevante no contexto de políticas públicas em saúde, principalmente quanto ao tema estudado.

\section{Resultados e Discussão}

A Região de Saúde de Piracicaba compreende 25 municípios, totalizando 1.429.471 habitantes, sendo que, desse total, 711.187 são do sexo masculino.

Embora se saiba que as internações por transtornos mentais (morbidade hospitalar) representam tão somente uma parcela do total dos casos existentes, esse dado representa a situação de saúde da população e pode dar base para a programação e gestão da saúde mental da população $^{(14)}$.

Analisando o número de internações realizadas pelo Capítulo V (transtornos mentais e comportamentais) da CID 10, observa-se expressivo aumento não linear nos últimos cinco anos, nos hospitais psiquiátricos de referência. Essa tendência é contrária à Lei Paulo Delgado (10.216/2001) e à Portaria GM/MS n².197, de 14 de outubro de 2004, que rege "VII - evitar a internação de usuários de álcool e outras drogas em hospitais psiquiátricos", possivelmente pela falta de outros recursos para o tratamento na especialidade $^{(15)}$.

Nota-se, também, que os transtornos mentais e comportamentais, referentes ao uso de álcool e outras drogas, representaram, desde 2003, a maior porcentagem do número total de internações pelo Capítulo $\mathrm{V}$ (transtornos mentais e comportamentais) da CID 10, indicando necessidade real de ações em saúde pública para prevenção e tratamento extra-hospitalar para esse subgrupo de doenças.

Além disso, dentre todas as internações por uso de substâncias psicoativas na Região de Saúde estudada, o consumo de álcool representa a maior porcentagem como mostra a Figura 1, ainda que inferior aos 90\% apresentados para o Brasil ${ }^{(13)}$. Porém, desde 2003, essa porcentagem tem diminuído, mostrando que o consumo de outras drogas está aumentando e levando a maiores complicações como a internação.

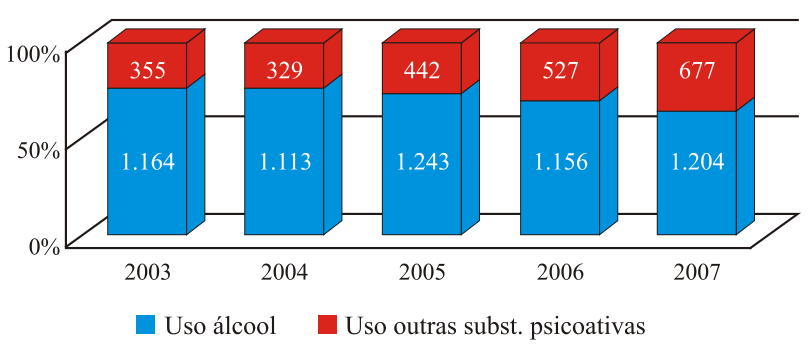

Figura 1 - Representatividade de internações entre os transtornos mentais e comportamentais, devido ao uso de todas as substâncias psicoativas, no período de 2003 a 2007

Fonte: SIH - Datasus - julho de 2008 
Os transtornos mentais e comportamentais face ao uso de substâncias psicoativas (F10 - F19 - CID 10) são responsáveis por $77,26 \%$ dos transtornos mentais como causa de morte no Brasil e, respectivamente, 59,11 e $53,38 \%$, no Estado e no município de São Paulo. Já na Regional de Saúde estudada, esse número é de $76 \%$, principalmente devido ao uso de substâncias psicoativas dentre os óbitos por transtornos mentais ${ }^{(14)}$.

Para atender essa demanda, a Região de Saúde possui dois hospitais cadastrados pelo Cadastro Nacional de Estabelecimentos de Saúde (CNES), na especialidade psiquiatria, sendo um no município de Araras, com 808 leitos disponíveis (639 SUS e 169 particulares) e um em Rio Claro, com 201 leitos (195 SUS e 6 particulares). Ambos são entidades filantrópicas de Gestão Estadual, classificados como de Atenção em Média Complexidade, que atendem demanda espontânea e referenciada. Nenhum deles possui diferenciação por equipes especializadas em dependência álcool e drogas, bem como não são cadastrados leitos específicos para esse tipo de tratamento.

Esses hospitais são os que dão o maior suporte para o atendimento hospitalar em Dependência Química, porém, foram constatadas internações em outras nove cidades.

Já no âmbito ambulatorial, especificamente para tratamento de dependência química, há um CAPS-AD localizado na cidade de Rio Claro e um Serviço Hospitalar de Referência, com 5 leitos, situado em Capivari, ambos inaugurados no ano 2007.

Tendo em vista o planejamento da cobertura da rede extra-hospitalar, as cidades da Região de Saúde que, de acordo com a Portaria GM 816, podem instituir CAPS-AD são: Araras, Limeira, Piracicaba e Rio Claro, respectivamente, apresentam 3, 0,7, 1 e 1,2 internações para cada mil habitantes, conforme Tabela 1 , tornando-se Piracicaba a mais indicada para implantação.

Tabela 1 - Internações por mil habitantes por município da Região de Saúde de Piracicaba

\begin{tabular}{|c|c|c|c|}
\hline Município & Internação & População & $\begin{array}{l}\text { Internação por } \\
\text { mil habitantes }\end{array}$ \\
\hline Águas de São Pedro & 3 & 2.039 & 1,5 \\
\hline Analândia & 10 & 4.061 & 2,5 \\
\hline Araras & 361 & 118.436 & 3,0 \\
\hline Capivari & 61 & 47.637 & 1,3 \\
\hline Charqueada & 29 & 14.996 & 1,9 \\
\hline Conchal & 54 & 25.572 & 2,1 \\
\hline Cordeirópolis & 33 & 21.207 & 1,6 \\
\hline Corumbataí & 6 & 4.338 & 1,4 \\
\hline Elias Fausto & 28 & 15.811 & 1,8 \\
\hline Engenheiro Coelho & 29 & 13.039 & 2,2 \\
\hline Ipeúna & 1 & 5.736 & 0,2 \\
\hline Iracemápolis & 20 & 18.791 & 1,1 \\
\hline Itirapina & 23 & 15.290 & 1,5 \\
\hline Leme & 159 & 9.1429 & 1,7 \\
\hline Limeira & 198 & 284.165 & 0,7 \\
\hline Mombuca & 3 & 3.544 & 0,8 \\
\hline Piracicaba & 385 & 372.073 & 1,0 \\
\hline Pirassununga & 89 & 71.772 & 1,2 \\
\hline Rafard & 10 & 8.166 & 1,2 \\
\hline
\end{tabular}

Continua...
Tabela 1 - Continuação

\begin{tabular}{lccc}
\hline \multicolumn{1}{c}{ Município } & Internação & População & $\begin{array}{c}\text { Internação por } \\
\text { mil habitantes }\end{array}$ \\
\hline Rio Claro & 226 & 193.719 & 1,2 \\
Rio das Pedras & 48 & 2.7230 & 1,8 \\
Saltinho & 4 & 6.413 & 0,6 \\
Santa Cruz da Conceição & 1 & 4.035 & 0,2 \\
Santa Gertrudes & 11 & 20.516 & 0,5 \\
Santa Maria da Serra & 23 & 4.991 & 4,6 \\
São Pedro & 67 & 34.465 & 1,9 \\
Total & 1.882 & 1.429 .471 & 1,3 \\
\hline
\end{tabular}

A proporção de CAPS-AD é inferior a 0,1 unidades por 10.000 habitantes, porém, não foi encontrado referencial comparativo de cobertura para a especialidade estudada.

Quanto à qualidade da assistência prestada nesses serviços, um indicador existente na fonte de pesquisa é o de tempo médio de permanência. Nota-se, na Tabela 2, que houve contínuo aumento na média de permanência nas internações dos hospitais de referência, que se estima estar em torno de 30 dias. Nas outras cidades em que ocorrem internações, os períodos de permanência foram menores que 5 dias.

Tabela 2 - Média de permanência por município e ano de competência, por local de internação, por transtorno mental, frente ao uso de substâncias psicoativas - Regional de Saúde Piracicaba - Ano 2003-2007

\begin{tabular}{lccccc}
\hline \multicolumn{1}{c}{ Município } & $\mathbf{2 0 0 3}$ & $\mathbf{2 0 0 4}$ & $\mathbf{2 0 0 5}$ & $\mathbf{2 0 0 6}$ & $\mathbf{2 0 0 7}$ \\
\hline Araras & $\mathbf{2 8 , 2}$ & $\mathbf{3 8 , 2}$ & $\mathbf{4 0 , 5}$ & 37,7 & 35,3 \\
Capivari & 1 & 1,5 & 3 & 2,5 & 1,8 \\
Conchal & 3,3 & 5 & 2 & 4 & 0 \\
Itirapina & 1,5 & 0 & 0 & 0 & 0 \\
Leme & 9 & 3,6 & 5,1 & 1 & 2,6 \\
Limeira & 4,3 & 4,5 & 2,4 & 1,8 & 0 \\
Piracicaba & 2,5 & 2,3 & 3,7 & 2,6 & 2,5 \\
Pirassununga & 2 & 2,5 & 2 & 2 & 7 \\
Rio Claro & 22,5 & 30,2 & 28,8 & 32,8 & 30,6 \\
Rio das Pedras & 2 & 3,3 & 2 & 0 & 0 \\
São Pedro & 0 & 0 & 2,1 & 0 & 0 \\
Total & 24,5 & 33,1 & 33,8 & 34,9 & 32,6 \\
\hline Fonte: SIH - Datasus - julho de 2008 & & & &
\end{tabular}

Nota-se, nessa informação, que o que é preconizado pela Portaria GM/MS n².197, de 14 de outubro de 2004, "VII - evitar a internação de usuários de álcool e outras drogas em hospitais psiquiátricos"(15) não está sendo seguido e está longe de corresponder às diretrizes de qualidade propostas para os Serviços Hospitalares de Referência, para a atenção integral aos usuários de álcool e outras drogas como:

"I - tratamento de intoxicação aguda, em Serviço Hospitalar de Referência para a Atenção Integral aos Usuários de Álcool e Outras Drogas - SHRad (tempo de permanência: 24 a 48 horas); II - tratamento da síndrome de abstinência do álcool, em SHRad (tempo de permanência: 3 a 7 dias); e III - tratamento de dependência do álcool, 
com a presença de intoxicação aguda com evolução para a instalação de síndrome de abstinência grave, ou ainda outros quadros de síndrome de abstinência seguidos por complicações clínicas, neurológicas e psiquiátricas, em SHRad (tempo de permanência: 3 a 15 dias)"(15).

Gastos com dependência química, como indica a Tabela 3, aumentaram aproximadamente $100 \%$ desde 2003, na Regional de Saúde de Piracicaba, mantendo aumento linear nas cidades em que há hospital de referência. Sendo que, na cidade de Araras, houve aumento de $100 \%$ e na cidade de Rio Claro aumento de $75 \%$.

Vale ressaltar que na cidade de Capivari, onde há leitos para internação em Hospital de Referência em Álcool e Drogas, o aumento de gastos foi de, aproximadamente, $400 \%$.

Tabela 3 - Valor total gasto em internações por transtornos mentais e comportamentais, face ao uso de substâncias psicoativas por local de internação, por município e ano de competência - Regional de Saúde de Piracicaba - Ano 2003-2007

\begin{tabular}{|c|c|c|c|c|c|}
\hline Município & $\begin{array}{c}2003 \\
R \$\end{array}$ & $\begin{array}{c}2004 \\
\mathrm{R} \$\end{array}$ & $\begin{array}{c}2005 \\
\mathrm{R} \$\end{array}$ & $\begin{array}{c}2006 \\
\mathrm{R} \$\end{array}$ & $\begin{array}{c}2007 \\
\mathrm{R} \$\end{array}$ \\
\hline Araras & $461.548,00$ & $668.361,38$ & $875.574,59$ & $870.857,05$ & $948.813,26$ \\
\hline Capivari & 118,83 & 410,58 & 143,25 & 265,30 & 942,11 \\
\hline Conchal & 331,47 & 450,54 & 127,35 & 99,11 & - \\
\hline Itirapina & 198,22 & - & - & - & - \\
\hline Leme & $2.292,90$ & $2.979,14$ & $1.379,80$ & 127,13 & $1.030,96$ \\
\hline Limeira & $4.979,19$ & $6.162,08$ & $4.375,84$ & 714,74 & - \\
\hline Piracicaba & $2.405,06$ & 446,25 & 995,49 & $4.803,08$ & $6.724,27$ \\
\hline Pirassununga & 99,11 & 243,53 & 167,73 & 127,35 & 386,81 \\
\hline Rio Claro & $519.814,84$ & $674.822,71$ & $718.171,91$ & $785.158,86$ & $829.947,83$ \\
\hline Rio das Pedras & 104,41 & 400,12 & 127,35 & - & - \\
\hline São Pedro & - & - & 757,89 & - & - \\
\hline Total & $991.892,03$ & $1.354 .276,33$ & $1.601 .821,20$ & $1.662 .152,62$ & $1.787 .845,24$ \\
\hline
\end{tabular}

Dessa forma, provavelmente, há demanda reprimida por não ter diminuído o valor gasto nas cidades com hospitais de referência, ou os casos são graves e estão sendo referenciados.

As informações de gastos ambulatoriais (repasse SUS) com dependência química da região estudada são recentes, sendo pouco representativos, pois há somente um CAPS-AD, o qual é referência. Além disso, ações dos serviços de atenção básica (PSF), realizadas para prevenção e assistência na especialidade estudada não estão contabilizadas nos gastos ambulatoriais, pois não há registros das mesmas.

Esses dados apontam para uma característica hospitalocêntrica da atenção em dependência química, na região estudada, pelo aumento no investimento em internações em serviços hospitalares puramente psiquiátricos e não na rede substitutiva.

Nota-se que os serviços de atenção à Saúde Mental e os investimentos nessa especialidade são baseados em internação em hospitais tradicionais, que, em sua história, veem o portador de transtorno mental em situação passiva e que deve ser isolado do convívio social. Essa prática deixa a desejar quando comparada às novas premissas de atenção integral que veem o paciente em sua forma biopsicossocial e têm como prioridade da política o CAPSAD como carro-chefe ${ }^{(8)}$.

\section{Conclusão}

Pode-se concluir que a Região de Saúde de Piracicaba, objeto deste estudo, necessita de mais investimento em saúde mental, principalmente na área de dependência química.

Os dados mostraram que há demanda, porém, poucos recursos ambulatoriais para prevenção e tratamento. Possivelmente, pacientes que poderiam ser tratados nessa modalidade estão sendo internados por falta de local de referência.

Há de ser lembrado que outros recursos contemplados no Programa de Atenção Integral a Usuários de Álcool e Outras Drogas, como parte da rede assistencial, não foram mapeados no estudo, como as associações Alcoólicos Anônimos (AA), Narcóticos Anônimos (NA), entre outros, mas devem ser considerados como parceria para o tratamento. Porém, não são em número suficiente para suprir a demanda que não é tratada ou acolhida pelo sistema governamental, nem devem ser os únicos serviços extra-hospitalares para assistência.

Além disso, para garantir assistência que contemple os princípios e diretrizes do SUS, a formação da rede assistencial deve ter em vista todos os recursos existentes em todos os municípios, e usar as ferramentas do sistema para planejamento e pactuação.

Faz-se necessária a adequação com as políticas do Ministério da Saúde, não só com relação à rede assistencial, mas, também, quanto à capacitação de Recursos Humanos para trabalhar nessa área.

As diversas complicações biopsicossociais desses pacientes tornam a assistência complexa e, além disso, para mudar a característica hospitalar, é necessário mudar a força de trabalho. Nesse sentido, outros trabalhos poderiam ser realizados para aprofundar quanto à qualidade assistencial e à capacitação contínua dos trabalhadores. 


\section{Referencias}

1. Berlinck MT, Magtaz AC, Teixeira M. A Reforma Psiquiátrica Brasileira: perspectivas e problemas. Rev Latino-Am Psicopatol Fund. 2008;11(1):21-7.

2. Amarante P. Novos sujeitos, novos direitos: o debate em torno da Reforma Psiquiátrica. Cad Saúde Pública. 1995;11(3):491-4.

3. Senna $M$ de CM. Eqüidade e política de saúde: algumas reflexões sobre o Programa Saúde da Família. Cad Saúde Pública. 200; 18:203-11. [acesso 19 set 2008]. Disponível em: http://www. scielo.br/scielo.php?script $=$ sci_arttext\&pid $=$ S0102311X2002000700020\&lng=pt\&nrm=iso.

4. Teixeira CF. Transição epidemiológica, modelodeatenção à saúde e previdência social no Brasil: problematizando tendências e opções políticas. Ciênc Saúde Colet. 2004;9(4). [acesso 19 set 2008]. Disponível em: http:// www.scielo.br/scielo.php?script=sci_arttext\&pid=S1413$81232004000400003 \& \operatorname{lng}=\mathrm{en} \& \mathrm{nrm}=$ iso.

5. Torre EHG, Amarante P. Protagonismo e subjetividade: a construção coletiva no campo da saúde mental. Ciênc Saúde Colet. 2001;6(1):73-85. [acesso 19 set 2008]. Disponível em: http://www. scielo.br/scielo.php?script $=$ sci_arttext\&pid=S1413$81232001000100006 \& \operatorname{lng}=\mathrm{en} \& n \mathrm{~nm}=$ iso.

6. Saraceno B. Reabilitação Psicossocial: uma estratégia para a passagem do milênio. In: Pitta A, organizador. Reabilitação Psicossocial no Brasil 1996. 2.ed. São Paulo: Hucitec; 1996.

7. Oliveira FB, Silva AO. Enfermagem em Saúde Mental no contexto da reabilitação psicossocial e da interdisciplinariedade. Rev Bras Enferm. 2000;53(4):584-92.

8. Moraes M. O modelo de atenção integral à saúde para tratamento de problemas decorrentes do uso de álcool e outras drogas: percepções de usuários, acompanhantes e profissionais. Ciênc Saúde Colet. 2008;13(1):121-33.

9. Machado AR, Miranda PSC. Fragmentos da história da atenção à saúde para usuário de álcool e outras drogas no Brasil: da Justiça à Saúde Pública. História, Ciências, Saúde. 2007;14(3):801-21.

10. Ministério da Saúde (BR). Política do Ministério da Saúde para Atenção Integral aos Usuários de Álcool e Outras Drogas. 2004. [acesso 14 julho 2008]. Disponível em: http://portal.saude.gov.br .

11. Vieira DL, Ribeiro M, Romano M, Laranjeira RR. Álcool e adolescentes: estudo para implementar políticas municipais. Rev Saúde Pública. 2007;41(3). [acesso 15 julho 2008]. Disponível em: http://www. scielo.br/scielo.php? script $=$ sci_arttext\&pid $=$ S003489102007000300011\&lng=pt\&nrm=iso.

12. Pinho PH, Oliveira MA de, Almeida MM de. A reabilitação psicossocial na atenção aos transtornos associados ao consumo de álcool e outras drogas: uma estratégia possível? Rev Psquiatr Clín. 2008;35 supl $1: 82-8$.

13. Noto AR, Galduroz JCF. O uso de drogas psicotrópicas e a prevenção no Brasil. Ciênc Saúde Colet. 1999; 4(1). [acesso 15 julho 2008]. Disponível em: http://www.scielo. br/scielo.php?script $=$ sci_arttext\&pid $=$ S1413-8123199900 0100012\&lng=pt\&nrm=isso.

14. Laurenti R. As manifestações de sofrimento mental mais frequentes na comunidade. SMAD, Rev Eletr Saúde Mental Álcool Drog. (Ed. port.). [online] 2007. 3(2). [acesso 14 julho 2008]. Disponível em: http://pepsic.bvspsi.org.br/scielo.php?script=sci_arttext\&pid=S1806-6976 2007000200007\&lng=pt\&nrm $=\overline{\text { isso. }}$.

15. Ministério da Saúde (BR). Portaria GM/MS No 2.197, de 14 de outubro de 2004. [acesso 14 julho 2008]. Disponível em: http://fjg.rio.rj.gov.br/publique/media/ PORTARIA\%20MS\%202.197.doc.

\section{Como citar este artigo:}

Vinha, IR. Cenário da assistência em saúde mental/uso de substâncias psicoativas na região de saúde de Piracicaba, São Paulo, Brasil. SMAD, Rev. Eletrônica Saúde Mental Álcool Drog. (Ed. port.). jan-abr 2011 [acesso:

31. Disponível em:
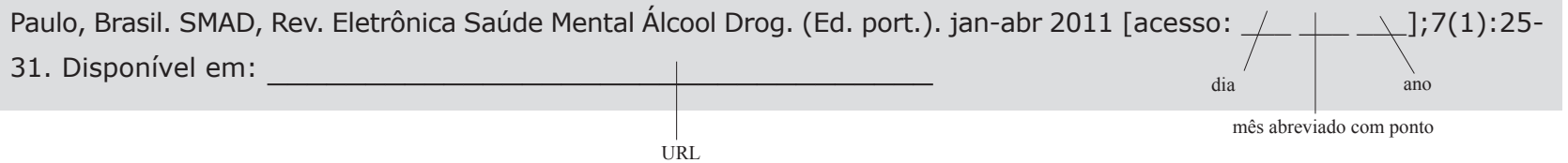